\title{
Einleitung: Die Corona-Pandemie - Praktische Philosophie in Ausnahmesituationen II
}

\section{Introduction: The Coronavirus pandemic - practical philosophy in exceptional circumstances II}

\section{Mark Schweda, oldenburg (im Namen aller Herausgebenden)}

Zusammenfassung: Die Corona-Krise gibt Anlass zu vielfältigen öffentlichen Debatten, die auch Fragen der Praktischen Philosophie berühren. Entsprechend intensiv fällt die philosophische Auseinandersetzung mit den verschiedenen Aspekten der Covid-19-Pandemie und ihrer individuellen und politischen Bewältigung aus. Dabei lassen sich im Verlauf des Pandemiegeschehens allerdings durchaus Verschiebungen der zentralen Themen, Positionen und Perspektiven beobachten.

Schlagwörter: Corona-Pandemie, Praktische Philosophie, Öffentlicher Diskurs

Abstract: The coronavirus crisis has sparked manifold public debates that also touch upon questions of practical philosophy. Hence an intensive philosophical discussion of the Covid-19 pandemic itself and the ensuing individual and political reactions is unfolding. At the same time, central topics, positions and perspectives are shifting in the course of the pandemic.

Keywords: coronavirus pandemic, practical philosophy, public discourse

Als wir im März 2020 den Call for Papers für einen Schwerpunkt der Zeitschrift für Praktische Philosophie zum Thema "Corona“ veröffentlichten, war die erste Welle der COVID-19-Pandemie gerade in Europa angelangt. In vielen Ländern traten weitreichende politische Maßnahmen zur Eindämmung der Ausbreitung des Erregers SARS-CoV-2 in Kraft. Die damit einhergehenden Einschränkungen des privaten und öffentlichen Lebens und ihre 
möglichen individuellen und sozioökonomischen Konsequenzen wurden zunehmend intensiv diskutiert.

Der Schwerpunkt „Corona-Pandemie - Praktische Philosophie in Ausnahmesituationen“, der schließlich im zweiten Heft des vergangenen Jahres - ungefähr auf dem Höhepunkt der zweiten Welle der Pandemie - in der Zeitschrift für Praktische Philosophie erschien, spiegelt diese Ausgangslage in vielen Hinsichten wider. Aus einer überwältigenden Gesamtzahl von beinahe 70 Einreichungen ausgewählt, erstreckte sich das thematische Spektrum der angenommenen Beiträge von den Anforderungen an klinische Ethikberatung im Zeichen von COVID-19 über die ethische Bedeutung der Selbstisolation für ein gutes Leben und die Auswirkungen des „Social Distancing“ auf leibkörperliche Nähe- und Distanzverhältnisse bis hin zur Erörterung von Fragen der lokalen Privatheit, geteilten Vulnerabilität und politischen Solidarität sowie der kritischen Auseinandersetzung mit den in der Corona-Krise zu Tage tretenden gesundheitsökonomischen Wertverständnissen und gesellschaftlichen Naturverhältnissen.

In der Redaktion beschäftigten uns seinerzeit zunächst durchaus grundsätzliche Fragen, die die Legitimation des Calls selbst und der aus ihm resultierenden Veröffentlichungen betrafen: Inwieweit war es gerechtfertigt und angemessen, auf eine in ihrem weiteren Verlauf und ihrem Ausgang noch vollkommen offene und unabsehbare Bedrohungslage, die zudem viele auf die ein oder andere Weise ganz unmittelbar vital und existenziell betraf, mit einem Aufruf zur Produktion und Publikation philosophischer Reflexionen zu reagieren? Wer hatte unter den gegebenen Bedingungen eigentlich die Zeit und Möglichkeit, sich an einem derartigen philosophischen Diskurs zu beteiligen (und wer nicht)? Und was hatte die Praktische Philosophie am Ende überhaupt zur Klärung oder gar Bewältigung einer Krise beizutragen, in der zunächst vor allem ganz „handfeste“ empirische Expertise aus Bereichen wie Virologie, Medizin und Gesundheitswissenschaften gefragt zu sein schien (Klonschinski 2020)?

In der Zwischenzeit hält der damals ausgerufene Ausnahmezustand nun bereits seit beinahe anderthalb Jahren an. Für viele zeichnen sich längst die Umrisse einer „neuen Normalität“ inmitten der Pandemie ab. Tatsächlich haben wir in vielen Hinsichten an Kenntnis, Übung und Routine im Umgang mit Corona gewonnen: Das Wissen über das Virus, seine Ausbreitung, die dadurch ausgelöste Erkrankung sowie ihre Präventions- und Behandlungsmöglichkeiten ist beträchtlich angewachsen. Die meisten haben sich an veränderte Alltagspraktiken und Umgangsformen ein Stück weit ge- 
wöhnt und in den durch den Infektionsschutz gesetzten Grenzen mehr oder weniger eingerichtet (oder gar Chancen in der Krise entdeckt). Die gesundheitlichen, psychischen und sozioökonomischen Folgekosten der Pandemie sind unterdessen deutlicher zu Tage getreten und werden wissenschaftlich eingehend vermessen und öffentlich kontrovers erörtert. Dabei hat auch eine große Anzahl an Veröffentlichungen aus Philosophie und angewandter Ethik zur Verständigung über die Lage beigetragen (vgl. allein für den deutschsprachigen Raum z.B. Demmerling 2021; Jaster und Keil 2021; Kröll et al. 2020; Mukerji und Mannino 2020).

Zugleich droht die Rede von der neuen Normalität und der Gewöhnung an den Ausnahmezustand jedoch darüber hinwegzutäuschen, dass wir uns nach wie vor in einer Situation befinden, die sich überaus dynamisch entwickelt. Die fortlaufende Veränderung des Pandemiegeschehens selbst sowie die damit einhergehenden politischen und gesellschaftlichen Nachjustierungen und Umstellungen setzen auch immer wieder neue Themen auf die Agenda, werfen andere Fragen auf und erfordern erneute Positionierungen. Wurde zu Beginn der Pandemie intensiv über den Zugang zu Beatmungsplätzen diskutiert, ist heute die Frage der Verteilung des inzwischen verfügbaren Impfstoffs sowie der rechtlichen Konsequenzen der Impfung in den Vordergrund gerückt. Standen anfangs vor allem ältere Menschen als Risikogruppe im Fokus, so werden mittlerweile auch verstärkt die Belange von Kindern, Jugendlichen und künftigen Generationen in den Blick genommen. Lag das Augenmerk zunächst vielfach auf den Herausforderungen, die die praktische Bewältigung der unmittelbaren Bedrohungslage mit sich brachte, so wendet sich der Blick inzwischen auch verstärkt zurück auf die Erfahrungen, die wir dabei als Individuen und Gesellschaft mit uns selbst gemacht haben und weiterhin machen. Es gibt erste Versuche einer vorgreifenden Bilanzierung dessen, was wir durch "Corona“ gelernt haben könnten für eine „postpandemische“ Zukunft.

Unter den Vorzeichen der augenscheinlich abflauenden dritten Welle ist die COVID-19-Pandemie auch im nun vorliegenden neuen Heft der Zeitschrift für Praktische Philosophie erneut Thema. Die eingegangenen, angenommenen und hier zu einem weiteren „Corona-Schwerpunkt“ zusammengefassten Beiträge beschäftigen sich ebenfalls mit ganz unterschiedlichen Aspekten des Pandemiegeschehens. Der Beitrag von Ronja Lutz (Erlangen), Andreas Frewer (Erlangen) und Cornelia Eibauer (München) wendet sich dem Bereich der Kinderpalliativmedizin und den hier erforderlichen Güterabwägungen in Zeiten von Corona zu. Die betroffenen Familien mit schwer- 
kranken Kindern werden im Kontext der COVID-19-Pandemie als eine vulnerable Gruppe aufgefasst, deren angemessene Versorgung diffizile individuelle und interindividuelle Abwägungen von Gütern wie Gesundheits- und Lebensschutz auf der einen und Lebensqualität, Freiheit und Partizipation auf der anderen Seite notwendig macht. Der Beitrag von Christian Hofmann (Hagen / Tutzing) erörtert im Anschluss die Bedeutung und Grenzen des Lebensschutzes als einer staatlichen Aufgabe. Dabei geht er insbesondere der Frage nach, inwieweit eine mit dem Lebens- und Gesundheitsschutz begründete Einschränkung individueller Freiheiten und gesellschaftlichen Lebens aus moral- und rechtsphilosophischer Sicht gerechtfertigt werden kann. Der Text von Sebastian Krach (Wien) unterzieht biopolitische Begrifflichkeiten und Perspektiven in Auseinandersetzungen über Regierungsmaßnahmen zur Eindämmung der Corona-Pandemie einer Kritik. Dabei führt er Michel Foucaults Verständnis von Biopolitik gegen die Auffassung (bio-)politischer Ordnungen bei Giorgio Agamben und Roberto Esposito ins Feld. Der Beitrag von Davina Höll (Tübingen) wendet sich schließlich den visuellen Darstellungen der Pandemie und den ethischen Implikationen ihrer Ästhetik zu. Ausgehend von den Bildern der Maske und des Virus selbst sowie ihren kulturhistorischen Tiefendimensionen geht er der Frage nach, wie Kunst und Literatur zu einer rhetorischen und metaphorischen Deeskalation pandemischer Krisendiskurse beitragen können.

\section{Literatur}

Demmerling, Christoph. 2021. „Schwerpunkt: Corona - eine Zwischenbilanz“. Deutsche Zeitschrift für Philosophie 69 (1): 25-28.

Jaster, Romy, und Geert Keil, Hrsg. 2021. Nachdenken über Corona. Stuttgart: Reclam.

Klonschinski, Andrea. 2020. „Philosophie in der Pandemie? Einleitung zum Schwerpunkt ,Die Corona-Pandemie - Praktische Philosophie in Ausnahmesituationen“'. Zeitschrift für Praktische Philosophie 7 (2): 245-252.

Kröll, Wolfgang, Johann Platzer, Hans-Walter Ruckenbauer und Walter Schaupp. 2020. Die Corona-Pandemie. Baden-Baden: Nomos.

Mukerji, Nikil, Adriano Mannino. 2020. Covid-19: Was in der Krise zählt. Über Philosophie in Echtzeit. Stuttgart: Reclam. 